\title{
A JSPSYCH PLUGIN FOR VISUAL ANALOGUE SCALES
}

\author{
A PREPRINT
}

\author{
Isaac Kinley \\ Department of Psychology, Neuroscience \& Behaviour \\ McMaster University \\ Hamilton, ON \\ kinleyid@mcmaster.ca \\ github.com/kinleyid
}

\begin{abstract}
Visual analogue scales (VASs) allow survey respondents to specify their answers with a high degree of precision, unlike Likert-type scales in which only a few categorical responses are available. In web-based research, HTML sliders (in which the respondent drags and drops a marker) are sometimes used as a substitute for VASs, but sliders produce data of a lower quality than true point-and-click VASs. Here, I introduce a plugin for jsPsych that enables survey responses using a true VAS, enabling researchers to collect self-report data on a continuum without the drawbacks of sliders.
\end{abstract}

\section{Introduction}

When collecting self-report data, researchers have several options. One is the Likert scale Likert [1932], in which participants select from a set of discrete options, each representing a value on some underlying continuum (e.g., agreement-disagreement). Because Likert scales do not produce continuous data, questionnaires using them should in theory be validated and analyzed using techniques for categorical data such as correspondence analysis [Greenacre. 2017] and polychoric correlation [Olsson, 1979]. However, techniques like these are relatively obscure among psychologists and their use is rare.

The visual analog scale (VAS; Hayes, 1921) is an alternative in which participants select a point on a line representing a continuum between two extremes (e.g., "least possible pain" to "worst possible pain"; Collins et al., 1997). Responses collected with VASs can be treated as interval data (or perhaps even ratio; Price et al., 1983, 2012), rather than nominal or ordinal, and are thus potentially amenable to parametric statistics [Dexter and Chestnut 1995]. While the dominant view is that the VAS produces ordinal data [Kim, 2017], its fine gradations are still an advantage over Likert scales. Indeed, Funke and Reips [2012] find that participants using digital VASs adjust their more responses more frequently than those using ordinal scales, suggesting increased care and more accurate responses. While administering VASs on paper is time-consuming, requiring researchers to manually measure the locations of participants' tick marks, this is not an issue with digital VASs used in online research.

A third option is the digital slider, in which the respondent clicks and drags a marker to any location along a continuum of values. HTML has a built-in "range" tool for sliders with basic support from all major browsers and the widely-used jsPsych software [De Leeuw , 2015] allows the use of sliders for recording responses to all of its supported categories of stimuli. In the context of web-based research, the history of sliders is different from Likert scales or VASs-rather than being invented by psychologists in a seminal experimental study of their use, sliders are simply a part of the HTML standard that has been adopted by researchers (myself included; Kinley et al., 2021) as an approximation of VASs. However, there are two important differences between sliders and VASs: first, sliders use a drag-and-drop mechanic in which the user must drag a marker to its final location rather than simply clicking that location; second, the slider marker must have a starting value from which it is subsequently dragged.

Both of these differences are consequential: Funke [2016] found that sliders, with their drag-and-drop mechanic, are uniquely difficult to use in online questionnaires (particularly on mobile devices) and suffer from lower response rates and higher response times than both Likert scales and point-and-click VASs. Similarly, Liu and Conrad [2019] and Maineri et al. [2021] find that responses made with a slider vary systematically based on the initial position of the slider 
Some people have the experience of finding themselves in a place and have no idea how they got there. Select the number to show what percentage of the time this happens to you.

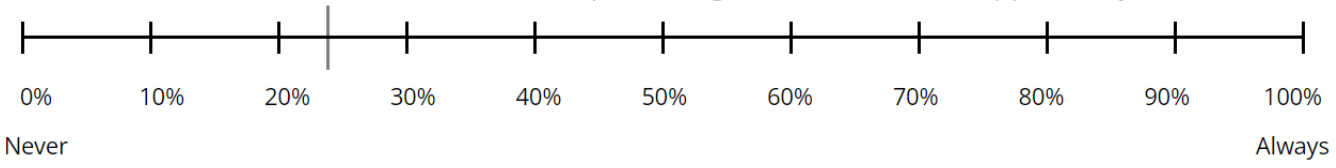

Continue

Figure 1: An example of the visual analog scale's appearance. The selected position shown produces a numerical value of 0.23875 , as the participant's click was 191 pixels from the left of the 800 pixel-wide scale. Question borrowed from the Dissociative Experiences Scale [Carlson and Putnam, 1993]

marker. In light of these issues, I introduce a jsPsych plugin for point-and-click visual analogue scales. My hope is for this tool to allow researchers using jsPsych to enjoy the benefits of a continuous measurement scale without the drawbacks inherent to sliders.

\section{Description of the tool}

Following jsPsych's naming conventions, the current tool is called jspsych-html-vas-response and is available from github.com/kinleyid/jspsych-html-vas-response. A demonstration of the tool can be found at kinleyid.github.io/rsrch/html-vas-response. It functions similarly to jspsych-html-slider-response, in that the experimenter specifies the stimulus displayed, the labels for the scale, and a prompt for the participant. The scale's appearance can also be customized, such as by adding tick marks or changing the colour of the scale and the appearance of the respondent's cursor when they hover over it (Fig. 1). The plugin records participants' responses as a number between 0 and 1 (where 0 is a click on the leftmost point of the line, 1 is a click on the rightmost point, and 0.5 is a click in the middle) as well as the response time (the time from the beginning of the trial to the participant clicking the "Continue" button) in milliseconds and the stimulus that was displayed. A full list of parameters and outputs is available in the tool's documentation on GitHub.

\section{References}

Rensis Likert. A technique for the measurement of attitudes. Archives of psychology, 1932.

Michael Greenacre. Correspondence analysis in practice. chapman and hall/crc, 2017.

Ulf Olsson. Maximum likelihood estimation of the polychoric correlation coefficient. Psychometrika, 44(4):443-460, 1979.

MH Hayes. Experimental developement of the graphics rating method. Physiol Bull, 18:98-99, 1921.

Sally L Collins, R Andrew Moore, and Henry J McQuay. The visual analogue pain intensity scale: what is moderate pain in millimetres? Pain, 72(1-2):95-97, 1997.

Donald D Price, Patricia A McGrath, Amir Rafii, and Barbara Buckingham. The validation of visual analogue scales as ratio scale measures for chronic and experimental pain. Pain, 17(1):45-56, 1983.

Donald D Price, Roland Staud, and Michael E Robinson. How should we use the visual analogue scale (vas) in rehabilitation outcomes? ii: Visual analogue scales as ratio scales: an alternative to the view of kersten et al. Journal of rehabilitation medicine: official journal of the UEMS European Board of Physical and Rehabilitation Medicine, 44(9):800, 2012.

Franklin Dexter and David H Chestnut. Analysis of statistical tests to compare visual analog scale measurements among groups. The Journal of the American Society of Anesthesiologists, 82(4):896-902, 1995.

Tae Kyun Kim. Practical statistics in pain research. The Korean journal of pain, 30(4):243, 2017. 
Frederik Funke and Ulf-Dietrich Reips. Why semantic differentials in web-based research should be made from visual analogue scales and not from 5-point scales. Field methods, 24(3):310-327, 2012.

Joshua R De Leeuw. jspsych: A javascript library for creating behavioral experiments in a web browser. Behavior research methods, 47(1):1-12, 2015.

Isaac Kinley, Morgan Porteous, Yarden Levy, and Suzanna Becker. Visual perspective as a two-dimensional construct in episodic future thought. Consciousness and Cognition, 93:103148, 2021.

Frederik Funke. A web experiment showing negative effects of slider scales compared to visual analogue scales and radio button scales. Social Science Computer Review, 34(2):244-254, 2016.

Mingnan Liu and Frederick G Conrad. Where should i start? on default values for slider questions in web surveys. Social Science Computer Review, 37(2):248-269, 2019.

Angelica M Maineri, Ivano Bison, and Ruud Luijkx. Slider bars in multi-device web surveys. Social Science Computer Review, 39(4):573-591, 2021.

Eve Bernstein Carlson and Frank W Putnam. An update on the dissociative experiences scale. Dissociation: progress in the dissociative disorders, 1993. 